\title{
DOCUMENTATION
}

\section{Les Ruptures de Barrages en terre.}

Dans cette imporlante étude aussi poussée que documentée d'illustrations et qui constitue une précieuse contribution à l'étude de ces ouvrages très spéciaux, l'auteur, après avoir exposé leur conceptoin générale, qui comporte généralement un diaphragme étanche formant "noyau " central interposé entre "2 massifs de terre ou matériaux de remblai arrangés par grosseur décroissante de l'extérieur vers l'intérieur, avec lalus perréyés, cite les acci- dents survenus aux plus importants de ceux de ces ouvrages qui ont cédé (Jonhson City, Tennessee; Davis Californie; Horse Creek, Colorado ; Mohawk, Ohio) ; s'efforçant d'en établir les causes et les responsabilités et d'en tirer, pour l'avenir. d'utiles enseignements.

J. B.

\section{Les nouveaux Transformateurs Américains de 20.000 KWA.}

Ces nouveaux engins sont conçus avec des perfectionnements non encore atteints à ce jour, pour pouvoir fonctionner avec la puissance indiquée, sous une tension variable entre 220 et $720.000 \mathrm{v}$. A ret effet, le refroidissement est assuré par une bonne circulation du licquide dans 18 tubes du radiateur, qui forment, atutour du transfo, une sorle de "faisceau "à très grande surface: et des dispositils spéciaux, interposés entre les éléments magné- ques et électriques, supportent les efforts mécaniques susceptibles de s'exercer entre les noyaux et le bobinage.

Le rendement (tout spécialement celui des appareils "autotransformateurs dans lesquels le secondaire fait partie du primaire) est très élevé et soutenu, atteignant, dépassant même. $99,50 \%$, malgré des pertes d'échauffement de l'ordre de plusieurs centaines de kwt. Power, 11 mars 1921. I. B.

\section{Les nouveaux Déversoirs à siphon.}

La question est déjà vieille, mais si elle a reçu diverses solutions ingénieuses, elle n'a encore point reçu de contribution capitale annulant les autres. Dans sa remarquable étude, M. Guido Ferrero en refait succinctement l'historique - attribuant la première conception à un ingénieur français, Girard - et décrit les principaux ouvrages de ce genre qui ont été construits et sont encore pour certains en service. Parmi ces ouvrages, il convient de citer le déversoir Grigotti, en béton armé; le système Vescovali -- en service à Rome; - celui de Heyn; du canal Oswego ; des Stadtwerke (Zurich); enfin, les systèmes, plus récents, de Vérone (canal Milani) et du canal Santa-Catarina. Nous ne pouvons évidemment que mentionner ces intéressants ouvrages, renvoyant, pour les détails, nos lecteurs aux textes originaux. J.B. Ann. dei $L_{\text {. L. Lubblici, avril } 1924 .}$

\section{Le Moteur asynchrone, reversible, à cage d'écureuil.}

Tout comme celui à rotor bobiné, le moteur alternatif, à cage d'écureuil peut, en lui imprimant une vitesse supérieure à sa vitesse de synchronisme, devenir générateur ; le réseau lui foumissant le courant nécessaire, le $\cos \phi$ en est influencé, sauf toutefois le cas où des condensateurs, aux bornes du stator, produiraient ce courant. EL clès lors, il devient possible, en raison du magnétisme rémanant du rotor, partant du repos, d'exciter la génératrice à cage, qui, simple et très robuste, se trouve particulièrement indiquée pour avoir sa place dans les petites exploitations isolées, branchées avec un réseau sur lequel elle peut débiter avec $\cos \vartheta=1$, et dans lesquelles elle constitue, de façon fort élégante, un parfait alternateur de secours ou de renfort.

J. B.

\section{L'Industrie Electrique, 25 janvier 1921.}

\section{La Régularisation dans les Usines hydro-électriques.}

Elle a été longtemps et est encore très rrécuemment assurée par des centrales de secours ou de réserve thermique. Cependant on cherche à les éviter - ou écarter - de plus en plus, en forçant sur l'acculumation de réserves hydrauliques lorsqu'elle est possible, ce cui n'est pas toujours le cas. L'auteur insiste sur l'intérêt qu'il y a généralement toujours à serrer de prìs le problème pour aboutir, dans bien des cas où les barrages sont impossibles, ou susceptibles de créer des réservoirs insuffisants, à utiliser, durant les périodes, de crues, la force supplémentaire disponible pour refouler l'eau dans un ou plusieurs bassins auxiliaires, d'altitude maxima, de façon à créer une chute artilicielle toujours disponible sous la main.

Il termine en citant de fort curieux et intéressants exemples, choisis dans les Alpes, parmi lesquels le plus marquant est celui des usines de Viverone (Italie), dans lesquelles $300.000 \mathrm{~m}^{3}$ travaillent sous $149 \mathrm{~m}$. de chute.

J. B.

\section{Relation entre la porosité et le facteur de puissance des isolateurs en porcelaine.}

J)es expériences et constatations auxquelles on procède depuis plusieurs années, spécialement en Amérique, il résulte que des isolateurs perdent, après leur mise en service, une partie de lcur résistance pouvant aller jusqu'aux $9 / 10^{\mathrm{e}}$, alors que certains autres, au contraire, de résistance moindre que les précédents, au sortir du lour, la maintiennent sensiblement constante, et que la diminution de résistance constatée est due à l'abśorption de l'humidité qui, pour" le courant H. T, constitue un chemin conducleur le long des conduits capillaires de porosité. En conséquence, nombre d'ingénieurs estiment aujourd'hui que la plus grande partie des détériorations des isolateurs en porcelaine qui paraissent de prime abord, inexplicables, proviennent de cette porosité. L'auteur de cet important article expose ses propres expériences pour éclairer la question, desquelles résulte nettement que, sous l'influence de l'humidité, lorsque la porcelaine est poreuse et, alors que la résistance diminue, lé facteur de charge augmente juscu'à atteindre et même dépasser $80 \%$; au contraire, lorsque la porcelaine n'est pas porcuse, facteur de puissance et porosité restent sensiblement constants. L'auteur explique ce phénomène par le fait que le courant qui traverse la porcelaine, en raison de l'humidité absorbée, est composant du courant total dont toute augmentation se traduit par une augmentation du facteur de puissance de l'isolateur (cos. ") quand il est soumis à un courant alternatif. J. B.

Rev. Un. des Mines, $1^{\text {er }}$ février 1924. 